$R M-75-49$

\title{
MEAN VALUE AND VARIABILITY OF FISH \\ CATCHES IN FLUCTUATING ENVIRONMENTS
}

M. Gatto

S. Rinaldi

September 1975

Research Memoranda are informal publications relating to ongoing or projected areas of research at IIASA. The views expressed are those of the authors, and do not necessarily reflect those of IIASA. 


\section{Mean Value and Variability of Fish

Catches in Fluctuating Environments

M. Gatto ${ }^{*}$ and S. Rinaldi**

\section{Abstract}

The mean value of the catch and its variability due to environmental fluctuations are analyzed for a very general stock-recruitment model. Particular attention is devoted to the comparison of two standard fishing strategies (constant effort and constant escapement) in terms of mean catch, variance in catches and maximum deviation of catch. It is demonstrated analytically that constant escapement policies should always give higher mean catch, but should also give higher catch variance and more extreme catches only under certain conditions of environmental variability.

\section{Introduction}

There is a need for fisheries management models and strategies that explicitly take environmental variability and unpredictable recruitment into account. Ricker [4] was perhaps the first to make a serious attempt to deal with this problem for single-aged fish stocks such as salmon. He compared two different fishing policies or "strategies" (cf. Allen, [1]), constant effort versus constant escapement. His main conclusions were that:

I The average catch obtained by fishing at constant escapement is greater than the one obtained by fishing at constant effort, and the difference between the two increases with the variability of the environment;

II The variability of the catch obtained in the case of constant escapement is greater than the one obtained in the case of constant effort.

* This work has been supported by the International Institute for Applied Systems Analysis (IIASA), Laxenburg, Austria. 
Recently, the problem has been more deeply investigated by Ricker and other authors (Larkin and Ricker, [3]; Tautz, Larkin and Ricker, [ 5 ]; Allen, [ 1 ]), whose work confirms properties I and II. Nevertheless, these properties have been "proved" only by means of extensive simulation, thus making explicit use of particular assumptions concerning noise (and the stock-recruitment curve). The aim of this paper is to prove the validity of property I under very general assumptions and then to point out that property II holds only under certain conditions, depending on whether variability is defined in terms of variance or of most extreme catches.

2. Description of the Model

Suppose a fishery is described by a discrete-time stock-recruitment relationship of the kind

$$
R_{t+1}=\alpha_{t} f\left(s_{t}\right)
$$

where $s_{t}$ is the number of spawners at time $t, R_{t+1}$ is the consequent recruitment and $\alpha_{t}$ is the growth factor which is assumed to be a stochastic process with mean value $\bar{\alpha}$ and variance $\sigma^{2}(\alpha)$. $f\left(s_{t}\right)$ is the underlying density dependent recruitment function, for example $s_{t} /\left(1+b s_{t}\right)$ in the well-known Beverton-Holt curve.

Eq. (1) with $S_{t}=R_{t}$ describes the natural evolution of the fishery, while the controlled evolution is described by Eq. (1) with

$$
s_{t}=R_{t}-c_{t}
$$

where the catch $c_{t}$ is a function $\phi$ of the recruitment

$$
c_{t}=\phi\left(R_{t}\right)
$$

Function $\phi$, called fishing policy or fishing strategy (Allen, [1]), can be specified by a curve lying in the cone $0 \leq \mathrm{C}_{t} \leq \mathrm{R}_{\mathrm{t}}$ of the catch-recruitment graph. The constant 
effort and constant escapement policies are shown in Figure 1 (curves (a) and (b) respectively). The point $(R, C)$ where the two curves intersect is assumed to correspond to a unique point on the "average" stock-recruitment curve $(R=\bar{\alpha} f(S))$. In other words, in a constant environment $\left(\alpha_{t}=\bar{\alpha}\right)$ the two policies would give rise to the same equilibrium. Thus the following relations among the parameters specifying the fishing policies hold:

$$
\begin{aligned}
& C=K R \\
& R=C+S \\
& S=(1-K) \bar{\alpha} f(S) .
\end{aligned}
$$

The following reasonable assumption on the "normalized" stockrecruitment curve $f$ and on the stochastic process $\alpha_{t}$ will be made:

Stock-recruitment function

i) $f(0)=0$

ii) $f^{\prime}(0)=1\left(f^{\prime}(S)\right.$ is the derivative of $f(S)$ )

iii) $f$ is bounded ( $f(S) \leq F)$

iv) $f$ is concave (bending over to the right). Stochastic process $\alpha_{t}$

v) $\alpha_{t}$ is a stationary and independent process

vi) $\alpha_{t}$ is bounded between two limits $A$ and $B\left(0 \leq A \leq \alpha_{t} \leq B\right)$.

All these assumptions except iv) are satisfied by classical stock-recruitment curves (the Ricker curve is not concave for high spawner levels). Nevertheless, real fisheries are usually such that the stock-recruitment curves are concave over the normal range of variability of the escapement (Cushing and Harris, [2]) and this is actually the property needed in the development which follows.

\section{Mean Value of the Catch}

Property I of Section 1 is now proved to hold under assumptions i) through vi).

In the case of a constant effort policy, Eq. (3) can be 


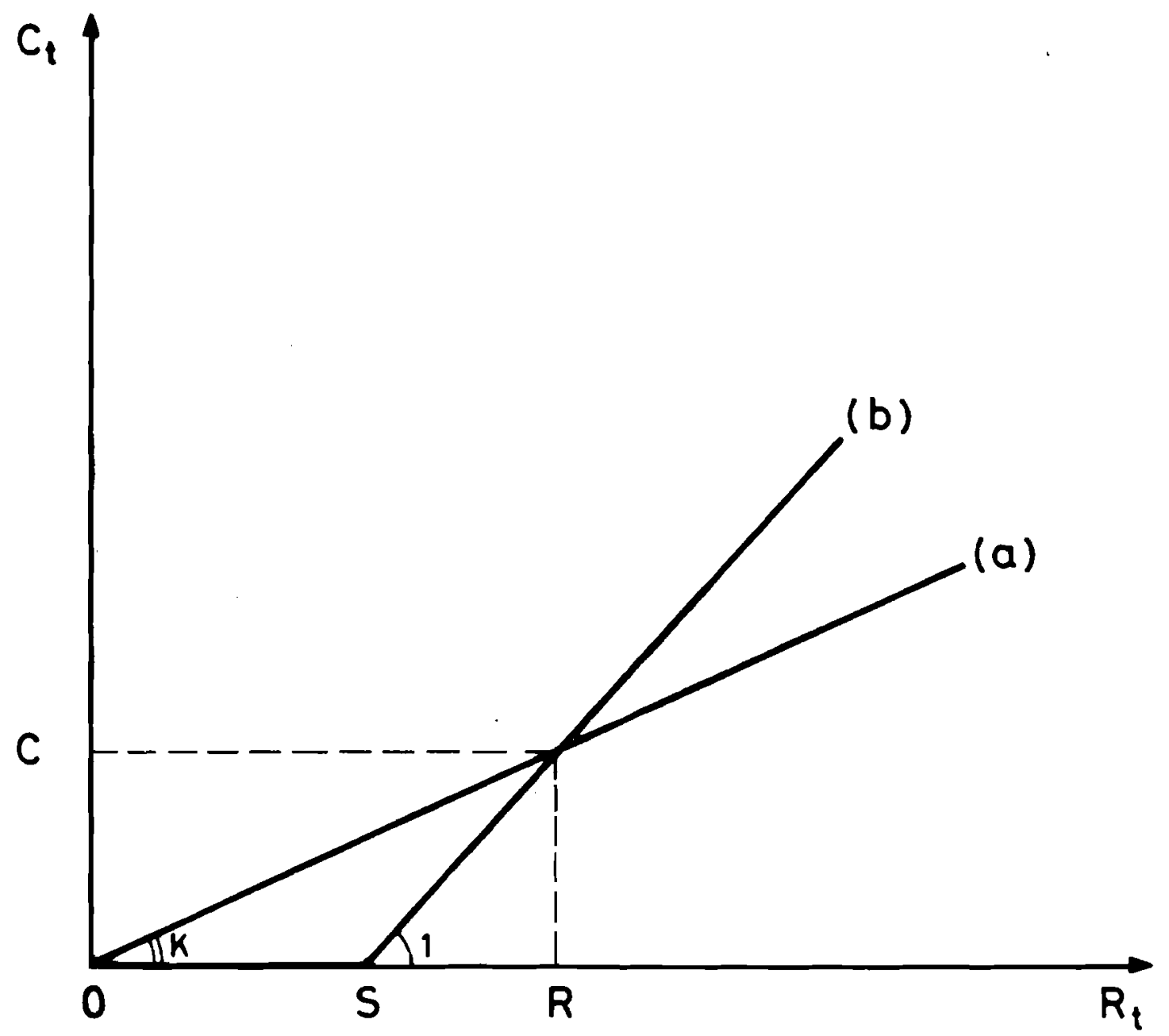

FIGURE 1. FISHING POLICIES: (a) CONSTANT EFFORT

(b) CONSTANT ESCAPEMENT 
specified as

$$
c_{t}=K R_{t}
$$

and the dynamics of the spawners described by

$$
s_{t+1}=\alpha_{t}(1-K) f\left(s_{t}\right)
$$

As far as the constant-escapement policy is concerned, a satisfactory approximation in the mean value of the catch is obtained by assuming that $s_{t}=s$ for all $t$. This is actually not the case whenever the size of the recruitment is less than the prescribed escapement $S$, a quite exceptional case indeed (Ricker, [ 4]; Larkin and Ricker, [ 3 ] ; Tautz, Larkin and Ricker, [5]; Allen, [1]). Moreover, it is worthwhile to notice that if the lower bound $A$ of the growth factor $\alpha_{t}$ is sufficiently high ( $A \geq S / \bar{I}(S)$ ), then the critical case $R_{t}<S$ never occurs. Thus, the catch at any time $t$ is given by

$$
c_{t}=\alpha_{t} f(S)-s
$$

and its average value $\overline{\mathrm{C}}_{\mathrm{S}}$ is given by

$$
\bar{c}_{S}=\bar{\alpha} f(S)-s
$$

Now, observe that Eqs. (2) and (7) imply $\bar{C}_{k}=(K / 1-K) \bar{S}_{k}$ while Eqs. (6) and (10) imply $\bar{C}_{S}=(K / 1-K) S$. Hence, in order to prove property I it only remains to verify that $\overline{\mathrm{S}}_{\mathrm{k}}<\mathrm{S}$. Thus, notice that Eq. (8) gives

$$
\overline{\mathrm{S}}_{\mathrm{K}}=\bar{\alpha}(1-\mathrm{K}) \overline{\mathrm{f}}_{\mathrm{K}}
$$

where $\bar{s}_{K}$ and $\bar{f}_{K}$ are the mean values of $s_{t}$ and $f\left(s_{t}\right)$ obtained by fishing at constant effort. Egs. (6) and (11) then give

$$
\frac{f(S)}{S}=\frac{\bar{f}_{K}}{\bar{S}_{K}}
$$


which implies

$$
\frac{f(S)}{S}<\frac{f\left(\bar{S}_{K}\right)}{\bar{S}_{K}} \text {, }
$$

because the function $f$ is concave. Finally, inequality and functions $i$ ) and $i v$ ) of $f$ imply $\bar{S}_{K}<S$ (and hence property I) .

It is important to note that this proof of property (1) gives some insight into the dependence of $\overline{\mathrm{C}}_{S}-\overline{\mathrm{C}}_{\mathrm{K}}$ upon the properties of the variability of the growth factor and the form of the stock-recruitment curve. In fact, for a given stockrecruitment curve the difference between $S$ and $\overline{\mathrm{S}}_{\mathrm{K}}$ (and hence between $\overline{\mathrm{C}}_{\mathrm{S}}$ and $\overline{\mathrm{C}}_{\mathrm{K}}$ ) will increase with $\sigma^{2}(\alpha)$ and, conversely, for a given stochastic process $\alpha_{t}$ the difference $s-\bar{s}_{K}$ will increase with the curvature of the stock-recruitment curve.

\section{Variance of the Catch}

In this section a precise meaning is given to the property of variability of the catch (property II of section 1). More precisely, it will be shown that for sufficiently high values of the variance $\sigma^{2}(\alpha)$ of the growth factor, the variance of the catch obtained by fishing at constant effort $\left(\sigma^{2}{ }_{K}(C)\right)$ is less than the one obtained by fishing at constant escapement $\left(\sigma^{2} s(C)\right)$.

Since in the constant-effort case

$$
c_{t+1}=K \alpha_{t} f\left(S_{t}\right)
$$

and $\alpha_{t}$ is an independent random variable, we obtain

$$
\varepsilon_{K}\left(C^{2}\right)=K^{2} \varepsilon\left(\alpha^{2}\right) \varepsilon_{K}\left(f^{2}\right),
$$

where $\varepsilon_{K}\left(C^{2}\right), \varepsilon\left(\alpha^{2}\right)$ and $\varepsilon_{K}\left(f^{2}\right)$ are the mean values of $C_{t}^{2}, \alpha_{t}^{2}$ and $f^{2}\left(S_{t}\right)$. But, since $f$ is bounded by $F$, we obtain

$$
\sigma_{\mathrm{K}}^{2}(C)<\varepsilon_{\mathrm{K}}\left(C^{2}\right)<\mathrm{K}^{2} \mathrm{~F}^{2}\left(\sigma^{2}(\alpha)+\bar{\alpha}^{2}\right)
$$


As for the constant escapement policy it is straightforward to show that

$$
\sigma_{S}^{2}(C)=f^{2}(S) \sigma^{2}(\alpha)
$$

Eqs. (14) and (15) allow us to show that for sufficiently high values of $\sigma^{2}(\alpha)$, i.e. for

$$
\sigma^{2}(\alpha)<\bar{\alpha}^{2} \frac{C^{2}}{R^{2}\left(\frac{R}{F}\right)^{2}-C^{2}}
$$

the variance of the catch is greater in the constant escapement case (see Fig. 2). Of course, this statement does not a priori imply that for low values of $\sigma^{2}(\alpha)$ the converse inequality holds for $\sigma_{K}^{2}(\alpha)$ and $\sigma_{S}^{2}(\alpha)$.

\section{Maximum Variation of the Catch}

This section is devoted to somehow reversing the conclusions of the previous section. It will be shown that if one considers the maximum variation of the catch from the nominal value $C$ as a variability index, then there are cases in which the constant effort policy implies a greater variability than the constant escapement policy.

To be more precise, define the two following indices of maximum variability

$$
\begin{aligned}
\Delta C^{+} & =\sup _{\alpha_{t, t}}\left(C_{t}-C\right) \\
\Delta C^{-} & =\sup _{\alpha_{t, t}}\left(C-C_{t}\right) .
\end{aligned}
$$

$c_{t}$ is the catch obtained by using a particular fishing policy with initial condition

$$
\mathrm{C}_{0}=\mathrm{C} ;
$$

$\Delta \mathrm{C}_{\mathrm{S}}^{+}, \Delta \mathrm{C}_{\mathrm{S}}^{-}$and $\Delta \mathrm{C}_{\mathrm{K}^{\prime}}^{+} \Delta \mathrm{C}_{\mathrm{K}}^{-}$are the indices for constant-escapement and constant-effort policies, respectively. 


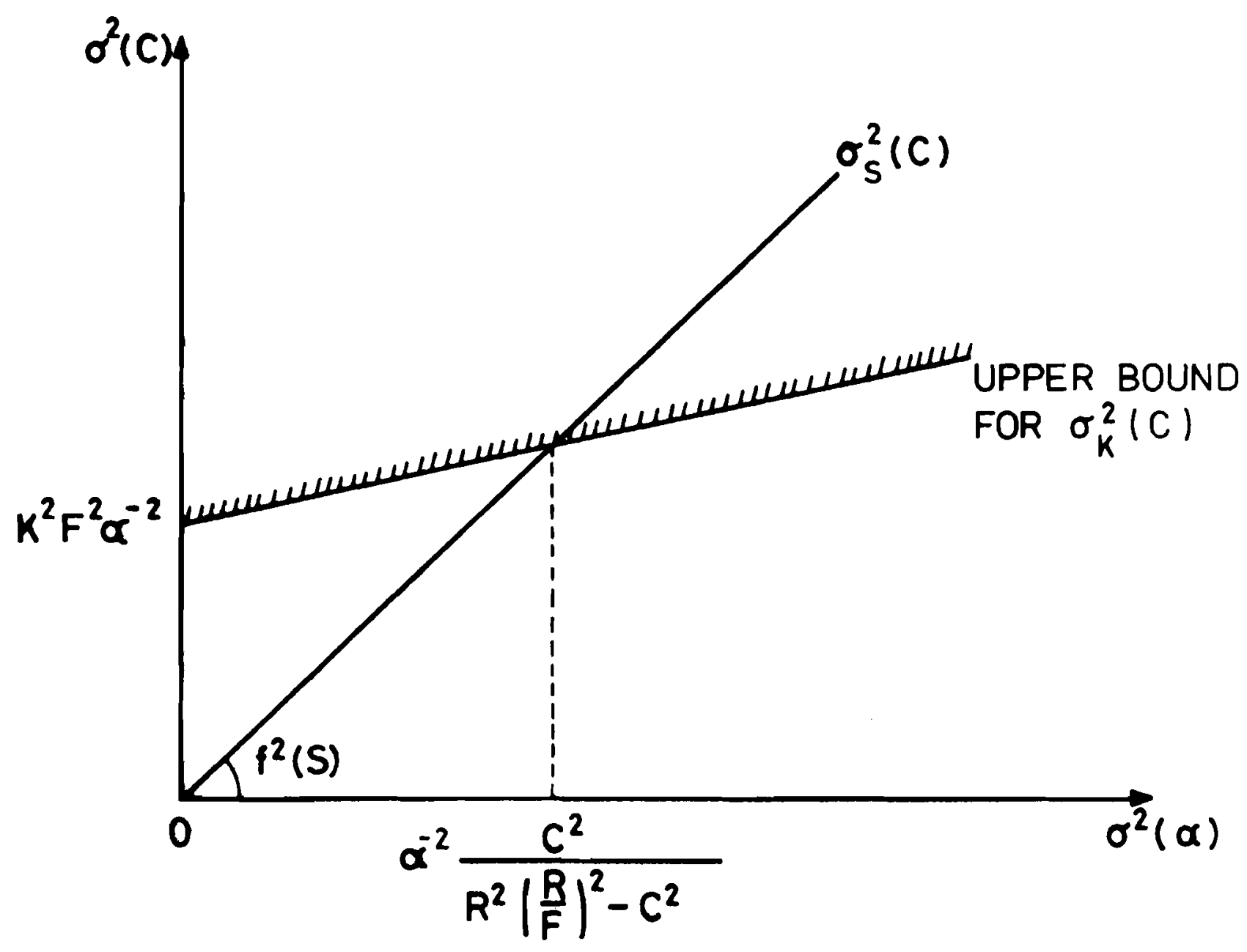

FIGURE 2. THE VARIANCE OF THE CATCH VS. THE VARIANCE OF THE GROWTH FACTOR 
In order to compare $\Delta \mathrm{C}_{\mathrm{S}}^{+}$with $\Delta \mathrm{C}_{\mathrm{K}}^{+}$and $\Delta \mathrm{C}_{\mathrm{S}}^{-}$with $\Delta \mathrm{C}_{\mathrm{K}}^{-}$, some relations between these indices and

$$
\begin{aligned}
& \delta \alpha^{+}=B-\bar{\alpha} \\
& \delta \alpha^{-}=\bar{\alpha}-A
\end{aligned}
$$

are first derived (note definitions of $A$ and $B$ in $v i$ ) above).

For the constant escapement policy this is achieved very easily, since Eqs. $(4-6)$ imply

$$
C=\bar{\alpha} f(S)-S,
$$

which, taking Eq.(9) into account, gives

$$
\Delta C_{S}^{+}=f(S) \delta \alpha^{+}
$$

Similarly,

$$
\Delta \mathrm{C}_{\mathrm{S}}^{-}=\mathrm{f}(\mathrm{S}) \delta \alpha^{-}
$$

i.e. $\Delta \mathrm{C}_{\mathrm{S}}^{+}$and $\Delta \mathrm{C}_{\mathrm{S}}^{-}$are linear functions of $\delta \alpha^{+}$and $\delta \alpha^{-}$.

For the constant effort policy, the analysis is unfortunately more cumbersome. First, observe that from Eqs. $(2,7,13)$

$$
c_{t+1}=K \alpha_{t} f\left(\frac{1-K}{K} c_{t}\right) \quad\left(c_{o}=c\right),
$$

and define $\mathrm{C}_{t}^{+}$and $\mathrm{C}_{t}^{-}$in the following way:

$$
\begin{array}{ll}
C_{t+1}^{+}=\operatorname{KBf}\left(\frac{1-K}{K} C_{t}^{+}\right) & \left(C_{O}^{+}=c\right) \\
C_{t+1}^{-}-\operatorname{KAf}\left(\frac{1-K}{K} c_{t}^{-}\right) & \left(c_{0}^{-}=c\right) .
\end{array}
$$

Thus, it is easy to prove that

$$
c_{t}^{-} \leq c_{t} \leq c_{t}^{+}
$$


for any $t$ and for any $\alpha_{t^{\prime}}$, provided that the function $f$ is an increasing function over the range of variability of the spawners, a condition which apparently is usually satisfied in real fisheries. In fact,

$$
C_{t+1}^{+}-C_{t+1}=K\left[B f\left(\frac{1-K}{K} C_{t}^{+}\right)-\alpha_{t} f\left(\frac{1-K}{K} C_{t}\right)\right],
$$

where $B \geq \alpha_{t}$ and $f$ is increasing. Thus $c_{t}^{+}-c_{t} \geq 0$ implies that the same condition is satisfied at time $t+1$, and similarly, $c_{t}-c_{t}^{-} \geq 0$ implies that $c_{t+1}-c_{t+1}^{-} \geq 0$. since $c_{0}^{+}-c_{0}=0$ and $c_{O}-c_{O}=0, E q$. (18) follows.

Now, call $\mathrm{C}^{+}$and $\mathrm{C}^{-}$the equilibrium catch for $\alpha_{t}=\mathrm{B}$ and $\alpha_{t}=A$, respectively; i.e.,

$$
\begin{aligned}
& c^{+}=\operatorname{KBf}\left(\frac{1-K}{K} c^{+}\right) \\
& c^{-}=\operatorname{KAf}\left(\frac{1-K}{K} c^{-}\right)
\end{aligned}
$$

Then,

$$
\begin{array}{lll}
\lim _{t \rightarrow \infty} c_{t}^{+}=c^{+} & \text {and } & c_{t}^{+}<c^{+} \\
\lim _{t \rightarrow \infty} c_{t}^{-}=c^{-} & \text {and } & c_{t}^{-}>c^{-},
\end{array}
$$

because $f$ is an increasing function. The conclusion of this discussion is that

$$
\begin{aligned}
& \mathrm{C}+\Delta \mathrm{C}_{\mathrm{K}}^{+}=\mathrm{K}\left(\bar{\alpha}+\delta \alpha^{+}\right) \quad \mathrm{f}\left(\frac{1-\mathrm{K}}{\mathrm{K}}\left(\mathrm{C}+\Delta \mathrm{C}^{+}\right)\right) \\
& \mathrm{C}-\Delta \mathrm{C}_{\mathrm{K}}^{-}=\mathrm{K}\left(\bar{\alpha}-\delta \alpha^{-}\right) \quad \mathrm{f}\left(\frac{1-\mathrm{K}}{\mathrm{K}}\left(\mathrm{C}-\Delta \mathrm{C}^{-}\right)\right),
\end{aligned}
$$

which can be solved with respect to $\delta \alpha^{+}$and $\delta \alpha^{-}$. Thus the derivatives of $\Delta \mathrm{C}_{\mathrm{K}}^{+}$and $\Delta \mathrm{C}_{\mathrm{K}}^{-}$with respect to $\delta \alpha^{+}$and $\delta \alpha^{-}$can be computed as 


$$
\begin{aligned}
& \frac{\mathrm{d} \Delta \mathrm{C}_{\mathrm{K}}^{+}}{\mathrm{d} \delta \alpha^{+}}=\left(\frac{\mathrm{d} \delta \alpha^{+}}{\mathrm{d} \Delta \mathrm{C}_{\mathrm{K}}^{+}}\right)^{-1} \\
& \frac{\mathrm{d} \Delta \mathrm{C}_{\mathrm{K}}^{-}}{\mathrm{d} \delta \alpha^{-}}=\left(\frac{\mathrm{d} \delta \alpha^{-}}{\mathrm{d} \Delta \mathrm{C}_{\mathrm{K}}^{-}}\right)^{-1}
\end{aligned}
$$

while the analogous derivatives for the constant-escapement case can be easily obtained from Eqs. $(16,17)$. A comparison of the two policies for values of $A$ and $B$ close to $\bar{\alpha}$ is now possible by evaluating these derivatives at $\delta \alpha^{+}=\delta \alpha^{-}=0$, thus obtaining (by cumbersome algebra)

$$
\begin{aligned}
& \frac{\mathrm{d} \Delta \mathrm{C}_{\mathrm{S}}^{\overline{+}}}{\mathrm{d} \delta \alpha^{\overline{+}}} \mid \begin{array}{l}
\delta \alpha^{\mp}=0
\end{array} \\
& \frac{\mathrm{d} \Delta \mathrm{C}_{\mathrm{K}}^{\overline{+}}}{\mathrm{d} \delta \alpha^{\overline{+}}} \mid \begin{array}{c}
\mathrm{K} \\
\end{array}
\end{aligned}
$$

These two equations allow comparison of the maximum variations of the catch for different values of $\bar{\alpha} f^{\prime}(S)$, the tangent to the average stock-recruitment curve. If $\bar{\alpha}^{\prime}(S)<1$ (see point $P$ of Fig. 3), then the constant excapment policy gives rise to a higher variability of the catch because

$$
\begin{aligned}
& \frac{d C_{\mathrm{K}}^{\bar{\tau}}}{\mathrm{d} \delta \alpha^{\overline{+}}}\left|<\frac{\mathrm{d} \Delta \mathrm{C}_{\mathrm{S}}^{\overline{ }}}{\mathrm{d} \delta \alpha^{\bar{\tau}}}\right| \\
& \delta \alpha^{\mp}=0 \quad \delta \alpha^{ \pm}=0 .
\end{aligned}
$$




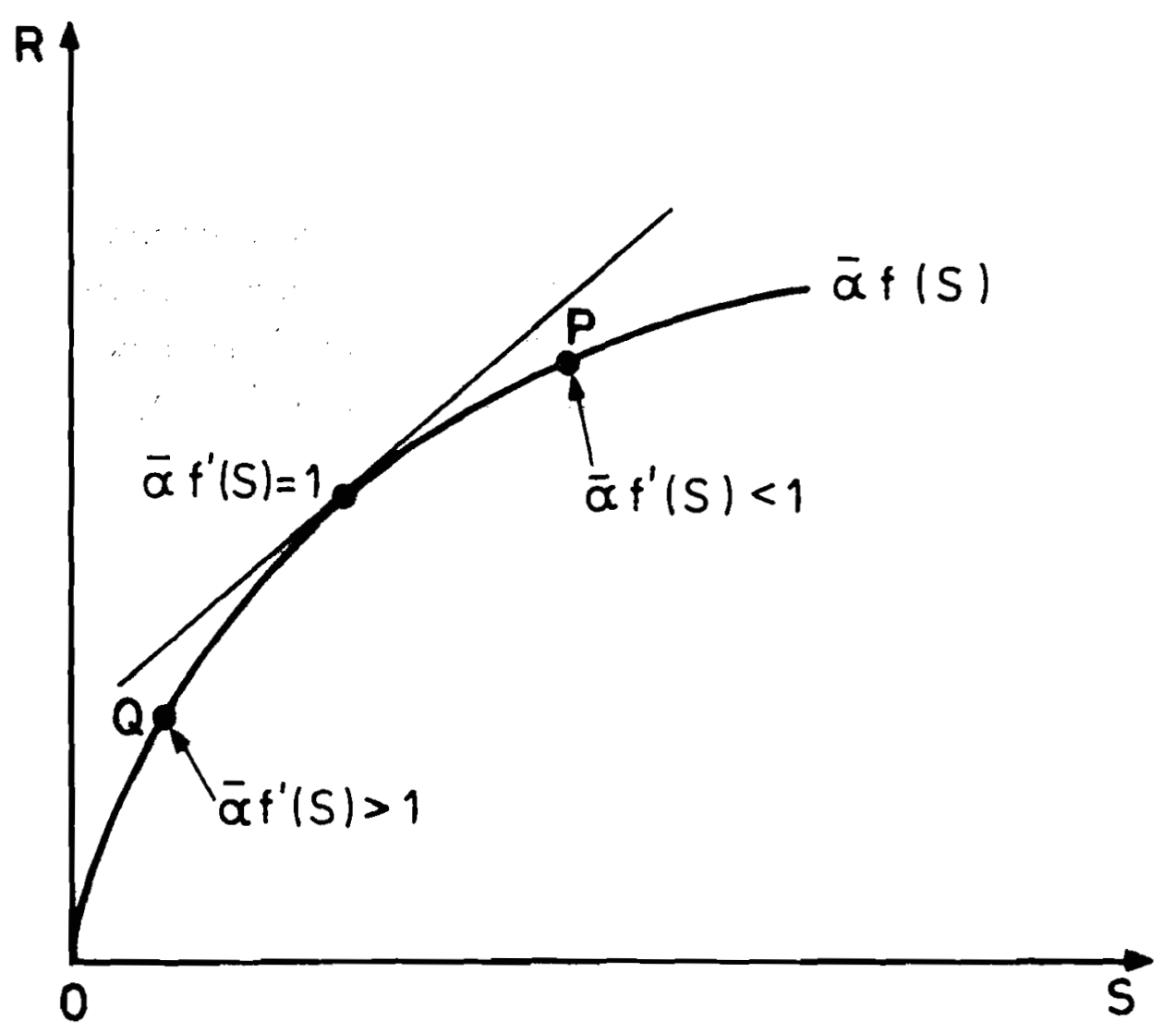

FIGURE 3. AVERAGE STOCK-RECRUITMENT CURVE AND MAXIMUM VARIATIONS OF THE CATCH: IN POINT P THE MAXIMUM VARIATION IS OBTAINED BY FISHING AT CONSTANT ESCAPEMENT; IN POINT Q THE MAXIMUM VARIATION IS OBTAINED BY :FISHING AT CONSTANT EFFORT 
But if $\bar{\alpha} f(S)>1$ (see point $Q$ of $\mathrm{Fig}$. 3), the higher maximum variation of the catch is obtained by means of the constant effort policy, a quite surprising result.

\section{Conclusion}

The aim of this paper has been to show general analytical conditions under which the numerical conclusions of Ricker and others are valid. We agree that the constant escapement policies should generally produce higher average catches, and that the price for higher averages will generally be higher variance of catches. However, the constant-effort and constant-excapement policies should produce similar extreme catches provided that the policies have been fixed on the basis of maximum sustained yield for the deterministic average.

\section{Acknowledgements}

The authors are deeply grateful to Dr. Carl Walters for his helpful criticism and encouragement. 
$\underline{\text { References }}$

[1] Allen, K.R. 1973. "The Influence of Random Fluctuations in the Stock-Recruitment Relationship on the Economic Return from Salmon Fisheries." Fish Stocks and Recruitment, edited by B.B. Parrish, P.A. Larkin, and W.E. Ricker, Conseil Int. pour l'Exploration de la Mer, Charlottenlund, Denmark.

[2] Cushing, D.H., and Harris, J.G.K. 1973. "Stock and Recruitment and the Problem of Density Dependence." Fish Stocks and Recruitment, edited by B.B. Parrish, P.A. Larkin, and W.E. Ricker, Conseil Int. pour l'Exploration de la Mer, Charlottenlund, Denmark.

[3] Larkin, P.A., and Ricker, W.E. 1964. "Further Information on Sustained Yields from Fluctuating Environments." J. Fish. Res. Bd. Canada, 21, $1: 1-7$.

[4] Ricker, W.E. 1958. "Maximum Sustained Yields from Fluctuating Environments and Mixed Stocks." J. Fish. Res. Bd. Canada, 15, 5 : 991-1006.

[5] Tautz, A., Larkin, P.A. and Ricker, W.E. 1969. "Some Effects of Simulated Long-Term Environmental Fluctuations on Maximum Sustained Yield." J.Fish. Res. Bd. Canada, 26, $10: 2715-2726$. 